\title{
New Pictures of the Structure and Plasticity of Orientation Columns in the Visual Cortex
}

\author{
Shigeru Tanaka
}

Additional information is available at the end of the chapter

http://dx.doi.org/10.5772/48396

\section{Introduction}

Orientation selectivity of neurons in the primary visual cortex is thought to be an important requisite for the preprocessing of visual information, which is followed by more complex information processing and representation in the extrastriate cortex for visual perception. It is widely accepted that neurons in the primary visual cortex optimally responding to similar stimulus orientations are clustered in a manner of straight columns extending from the superficial to deep layers (Hubel \& Wiesel, 1962, 1963a). The cerebral cortex is, however, folded inside a skull, which makes gyri and fundi. Particularly, in cats, area 17 (primary visual cortex) is located on the curved cortex called the lateral gyrus (Tusa et al., 1978). These facts raise questions of how the tangential arrangement of orientation columns is reconciled with the curvature of the gyrus, and whether the columns penetrate the cortex from the superficial to deep layers. In the first part of this chapter, we show a possible configuration of feature representation in the visual cortex using a three-dimensional (3D) self-organization model, and then confirm the predicted 3D orientation representation using multi-slice, high-resolution functional magnetic resonance imaging (fMRI) performed in the cat visual cortex (Tanaka et al., 2011). We obtained a close agreement in orientation representation between theoretical predictions and experimental observations. These studies demonstrated that in the curved cortex, preferred orientations are represented by wedgelike orientation columns which do not necessarily penetrate from superficial to deep layers, whereas in the flat cortex, preferred orientations are tended to be represented by classical straight columns.

On the other hand, numerous studies have been devoted to solving a question of how orientation selectivity is established and elaborated in early life. It is believed that orientation selectivity innately emerges prior to visual experience (Albus \& Wolf, 1984; 
Blakemore \& Van Sluyters, 1975; Crair et al., 1998; Hubel \& Wiesel, 1963b), but there has been a debate on the issue whether visual experience can modify orientation selectivity. Historically, Blakemore \& Cooper (1970) reported that orientation-restricted visual experience modified response properties of visual cortical neurons so as to respond selectively to experienced orientations, which suggests a validity of the instruction hypothesis: Preferred orientations of neurons shift to experienced orientations (Rauschecker \& Singer, 1981). Later, Stryker et al. (1978) and Carlson et al. (1986) claimed an objection against this hypothesis and proposed the suppression hypothesis: Neurons innately selective for inexperienced orientations only diminish their responsiveness while preferred orientations do not change. The current consensus of orientation plasticity in early life favors the suppression hypothesis. However, considering that visual experience for a few days under monocular deprivation shifts ocular dominance of visual cortical neurons towards an open eye (Wiesel \& Hubel, 1963), one may not feel that the selection hypothesis is convincing. In the second part of this chapter, we show data obtained from in vivo intrinsic signal optical imaging in the visual cortex of kittens reared with head-mounted cylindrical-lens-fitted goggles for stable exposure to a single orientation (Tanaka et al., 2004, 2006, 2007). It was revealed that single-orientation exposure expands the cortical territory that represents the exposed orientation, as a previous study by Sengpiel et al. (1999). However, the degree of overrepresentation of the exposed orientation was more prominent in our data due to several differences of visual experience manipulation. Also, we show an age-dependent sensitive period profile for orientation selectivity in the visual cortex of goggle-reared kittens (Tanaka et al. 2009).

\section{Theory and experiment on $3 \mathrm{D}$ orientation representation}

We employed a self-organization model with minimal assumptions to describe the formation of joint maps of the preferred orientation, preferred direction, ocular dominance and retinotopy. We performed computer simulation using a structural annealing: Simulation was started only in the middle layer at the beginning and then the simulation range was expanded gradually to other layers. This annealing method well reproduced orientation columns vertically spanning all the layers in the flat cortex, consistent with widely accepted columnar organization. On the other hand, in the curved parts, orientation straight columns were disrupted and preferred orientations were clustered in wedge-like forms. It was likely that preferred directions are reversed in the deeper layers. Singularities associated with orientation representation appeared as warped lines in the 3D model cortex. Direction reversal appeared on the sheets that were delimited by orientation-singularity lines. These structures emerged from the balance between periodic arrangements of preferred orientations and vertical alignment of the same orientations. Then, to examine the biological plausibility of the simulation results, we attempted to visualize orientation representation in the cat visual cortex using multi-slice, high-resolution fMRI. We obtained a close agreement in orientation representation between theoretical predictions and experimental observations (Tanaka et al., 2011). 


\subsection{Mathematical modeling}

We extended the previously reported model (Nakagama \& Tanaka, 2004) to describe the formation of orientation, direction, ocular dominance and retinotopic representation in the 3D primary visual cortex. For simplicity, among 6 layers of the visual cortex, we omitted layer 1, in which massive fibers are running tangentially and neurons exist sparsely. In the other 5 layers, we assumed that each neuron represents a priori a set of visual features such as ocular dominance, orientation preference, direction-of-motion preference and the center of the receptive field for simplicity. The cortical arrangement of the visual features was modified by local intracortical interaction among neurons, which change an initial random arrangement of visual features to form an orderly arrangement over the 3D primary visual cortex.

\subsubsection{Three-dimensional model visual cortex and visual feature space}

One of the purposes of this study is to investigate the effect of the global curvature of the visual cortex on visual feature representation. For this purpose, we assumed a "stadiumshape" of the model cortex with a finite thickness, which is embedded inside a box composed of $128 \times 128 \times 72$ voxels. Each voxel represents a cortical neuron. The outer and inner surfaces, respectively, correspond to the cortical surface and the boundary between the gray and white matter. The model cortical depth was set at 30 voxels with a 6 -voxel thickness of each layer from layer 2 to layer 6 . We assumed the thickness of the model cortex to be $2 \mathrm{~mm}$, meaning one voxel is $66 \mu \mathrm{m}$. The periodic boundary condition was adopted to minimize a small-size effect. The feature space (FS) is given by the direct product of the space of the visual field (VF), the circular symmetric space of the preferred direction (PD) and the space of ocular dominance (OD): $\mathrm{FS}=\mathrm{VF} \times \mathrm{PD} \times \mathrm{OD}$. Space VF is the $2 \mathrm{D}$ visual field $(26 \times 54$ grids), in which the position of a receptive field center of a cortical neuron is defined. The periodic boundary condition was imposed on space VF so as to be consistent with the periodic boundary condition imposed on the model visual cortex. The preferred orientation is defined as the axis orthogonal to the preferred direction of motion. The space OD is subdivided into five groups, in which groups 1 and 5 are driven exclusively by either of the two eyes, group 3 is driven by the two eyes equally, and groups 2 and 4 receive imbalanced inputs from the two eyes.

\subsubsection{Energy function}

We assume that a neuron located at $j$ in the model visual cortex represents a set of visual features $(k, \theta, \mu)$, where $k, \theta$ and $\mu$ indicate the position of the receptive field, preferred direction and ocular dominance, respectively. The ocular dominance $\mu$ takes a value out of $-1,-1 / 2,0,1 / 2$, and $1: \mu=1$ represents exclusively the left-eye dominance and $\mu=-1$ exclusively the right-eye dominance. The preferred direction $\theta$ takes one of 16 values out of $0, \pi / 8,2 \pi / 8, \ldots$, and $15 \pi / 8$. The preferred orientation was assumed to be orthogonal to the preferred direction and invariant under rotational transformation by $\pi$. When a cortical neuron $j$ represents $(k, \theta, \mu), \sigma_{j, k, \theta, \mu}$ takes 1 , and otherwise, 0 . Here we assume that any 
cortical neuron is supposed to represent only one set of visual features. The state of the visual feature arrangements is characterized by the energy function:

$$
H=-\sum_{j, j^{\prime}} \sum_{k, \theta, \mu} \sum_{k^{\prime}, \theta^{\prime}, \mu^{\prime}} V_{j, j^{\prime}} \Gamma_{k, \theta, \mu ; k^{\prime}, \theta^{\prime}, \mu^{\prime}} \sigma_{j, k, \theta, \mu} \sigma_{j^{\prime}, k^{\prime}, \theta^{\prime}, \mu^{\prime}}+c \sum_{l=2}^{6} \sum_{k, \theta, \mu}\left(\sum_{j \in l \text {-th layer }} \sigma_{j, k, \theta, \mu}\right)^{2},
$$

where $V_{j, j^{\prime}}$ represents the 3D cortical interaction between a pair of visual features represented by neurons $j$ and $j^{\prime}$. This interaction is determined by the convolution of the dendritic arbor function with the axonal arbor function. It is know that in the adult cortex, there are longrange tangential and inter-layer connections. However, we assume that the axonal arbor of nearby cortical neurons mainly works for column formation in the early developmental stage, and we omit the long-range connections. This assumption indicates that the interaction does not depend on the global morphology of the cortex and is identical for any positions either in the superficial layers or deep layers. Therefore, we identify the cortical interaction as the dendritic integration, which is simply given by the Gaussian function:

$$
V_{j, j^{\prime}}=\frac{1}{2 \pi \lambda_{v}^{2}} \exp \left(-\frac{d_{j, j^{\prime}}^{2}}{2 \lambda_{v}^{2}}\right)
$$

where $\lambda_{v}$ is the excitatory interaction length in the cortex $\left(\lambda_{v}=2.0\right.$ corresponding to 132 $\mu \mathrm{m})$, and $d_{j, j^{\prime}}$ indicates the distance between cortical neurons $j$ and $j^{\prime} . \Gamma_{k, \theta, \mu ; k^{\prime}, \theta^{\prime}, \mu^{\prime}}$ represents the correlation function of input signals. The second term of Eq. (1) represents a constraint on the representation of the visual features to avoid imbalanced representation and results in each layer covering all the feature space as uniformly as possible. The constraint is imposed layer by layer. The sum of $j$ is taken over all neurons located within the $l$-th layer of the model cortex. The strength of constraint $c$ was set at 0.008 .

The correlation function of input signals $\Gamma_{k, \theta, \mu ; k^{\prime}, \theta^{\prime}, \mu^{\prime}}$ is modeled by assuming that local components of visual images obey white noise regarding the position and direction of motion with a strength of correlation between the two eyes $r$, and that the input signal receptive field is given by the product of the positional Gaussian function and the directional cosine series. After short calculations, the correlation functiont is rewritten as

$$
\Gamma_{k, \theta, \mu ; k^{\prime}, \theta^{\prime}, \mu^{\prime}}=S_{k, k^{\prime}} \Psi_{\theta, \theta^{\prime}} \mathrm{O}_{\mu, \mu^{\prime}}
$$

where $S_{k, k^{\prime}}, \Psi_{\theta, \theta^{\prime}}$ and $\mathrm{O}_{\mu, \mu^{\prime}}$ represent component correlation functions regarding the position of receptive field centers in the visual field, direction of motion and ocular dominance, respectively. These component correlation functions are given by

$$
S_{k, k^{\prime}}=\exp \left(-\frac{d_{k, k^{\prime}}^{2}}{2 \lambda_{\text {corr }}^{2}}\right)
$$




$$
\begin{gathered}
\Psi_{\theta, \theta^{\prime}}=2 a_{0}^{2}+\sum_{m=1}^{\infty} a_{m}^{2} \cos m\left(\theta-\theta^{\prime}\right), \\
\mathrm{O}_{\mu, \mu^{\prime}}=\frac{(1+r)+(1-r) \mu \mu^{\prime}}{2} .
\end{gathered}
$$

Here, $\lambda_{\text {corr }}$ is the positional correlation length equal to $\sqrt{2} \lambda_{\text {ref }}$, which is set at 1.0. The directional component of the correlation function is given as a Fourier cosine series. The order of $m=1$ represents the correlation between a pair of preferred directions, whereas the order of $m=2$ represents the correlation between a pair of preferred axes of motion, which are orthogonal to the preferred orientations.

\subsubsection{Computer simulation}

At the beginning of the simulation, we randomize visual features in the model cortex. In the middle layer of the cortex, neurons receive random inputs from either eye $(\mu=+1$ or -1$)$, because the layer is geniculocortical input layer. In the other layers, neurons receive random inputs with ocular dominance of either of $-1,-1 / 2,0,1 / 2$, or 1 , and preferred direction of either of $0, \pi / 8,2 \pi / 8, \cdots$, or $15 \pi / 8$. The RF center position is also randomized retaining rough retinotopy according to the probability:

$$
A_{k ; j}=\exp \left(-\frac{d_{J_{k} ; j}^{2}}{2 \lambda_{\text {Ret }}^{2}}\right),
$$

where $d_{J_{k} ; j}$ represents the distance between cortical position $j$ and position $J_{k}$ that retinotopically corresponds to the position $k$ in the visual field, where $\lambda_{\text {Ret }}$ represents the roughness of retinotopy $\left(\lambda_{\text {Ret }}=3.5\right)$. For each trial of updating visual features in the simulation, a new candidate for a set of visual features is selected according to the same probability distribution as in the initial randomization, and then the old set of features is replaced with the new one according to the probability determined by the energy difference between the states before and after the trial of replacement:

$$
\operatorname{Pr}(\text { before } \rightarrow \text { after })=\frac{1}{1+\exp \left[\beta\left(H^{\text {after }}-H^{\text {before }}\right)\right]} .
$$

Here, $H^{\text {before }}$ and $H^{\text {after }}$ represent the energy before and after the trial of replacement, respectively. The parameter $\beta$, which determines the steepness of the logistic function of the right-hand side of Eq. (8) in the transition region, was set at $\beta=\infty$. Thereby, the probability takes 1 when $H^{\text {after }}$ is smaller than $H^{\text {before }}$, otherwise 0 . The repetition of the update procedure likely decreases the energy of the system and realizes an equilibrium cortical arrangement of visual features when the change in energy becomes negligibly small.

In the present model, in which the cortical interaction function is isotropic, clustering of similar visual features occurs but columnar organization cannot be formed even in flat parts 
of the model visual cortex when the simulation is carried out to update features sampled randomly from the entire model cortex. So, we carried out the simulation according to the following schedule of spatial simulated annealing that we call hereafter, which assists the formation of iso-orientation domains continuously extending from layer 2 to layer 6 . For simulation step $t$ in the interval of $1 \leq t<T_{1}$, simulation was performed only in the middle layer whose upper and lower boundaries were located at 13 and 18 voxels from the bottom of the model cortex. For $T_{1} \leq t \leq T_{2}$, the inner boundary $z^{\text {inner }}$ and the outer boundary $z^{\text {outer }}$ of the simulation range in the depth direction was gradually changed as $z^{\text {inner }}=13-\left\lceil 12 \times \frac{t-T_{1}}{T_{2}-T_{1}}\right\rceil$, and $z^{\text {outer }}=18+\left\lceil 12 \times \frac{t-T_{1}}{T_{2}-T_{1}}\right\rceil$. For $T_{2}<t$, simulation was carried out in all model visual cortex. Here, one simulation step is defined as the trial numbers of updating features by the total number of voxels within which simulation is conducted. That is, for 1 simulation step, each voxel is tried to be updated, on average, once. Parameters $T_{1}$ and $T_{2}$ were set at 100 and 350 simulation steps. Thus, columnar structures of visual features are likely formed owing to the spatial simulated annealing, even if we do not assume anisotropic cortical interaction.

\subsubsection{Analyses of cortical feature representations}

An output response of neuron $j$ to the presentation of visual images through either eye is given by the integration of evoked activities at nearby neurons through the dendrite. Therefore, the output neuronal response is expressed by

$$
\eta_{j ; k, \theta, \mu}^{\text {out }}=\sum_{j^{\prime}, l, \varphi} V_{j ; j^{\prime}} \sigma_{j^{\prime} ; l, \varphi, \mu} R_{l, \varphi ; k, \theta} .
$$

Here we suppose that a global stimulus like an oriented grating stimulus moving in the direction $\theta$ is presented to both eyes. The direction tuning curve of model cortical neuron $j$ is defined by

$$
\varsigma_{j}^{\text {dir }}(\theta)=\sum_{k, \mu} \eta_{j ; k, \theta, \mu}^{\text {out }}
$$

The preferred direction of the neuron $j$ is determined when $\varsigma_{j}^{d i r}(\theta)$ takes the maximum at $\theta=\theta_{j}^{\text {pref }}$. The orientation tuning curve of model cortical neuron $j$ is defined by the sum of responses to opposite directions orthogonal to the stimulus orientation $\phi$ as follows:

$$
\zeta_{j}^{\text {ori }}(\phi)=\frac{1}{2} \sum_{k, \mu}\left(\eta_{j ; k, \phi-\pi / 2, \mu}^{\text {out }}+\eta_{j ; k, \phi+\pi / 2, \mu}^{\text {out }}\right)
$$

The preferred orientation of this neuron is determined when the orientation tuning curve $\zeta_{j}^{\text {ori }}(\phi)$ takes the maximum at $\phi=\phi_{j}^{\text {pref }}$. The ocular dominance of neuronal responses is defined by the contrast form of responses to the left- and right-eye stimuli as follows: 


$$
O_{j}=\frac{\sum_{k, \theta, \mu} \mu \eta_{j ; k, \theta, \mu}^{\text {out }}}{\sum_{k, \theta, \mu} \eta_{j ; k, \theta, \mu}^{\text {out }}} .
$$

To analyze orientation and direction singularities, we calculate an absolute value of the gradient vector for the preferred orientation, $\left|\vec{\nabla} \phi_{j}^{\text {pref }}\right|=\sqrt{\left(\partial_{x} \phi_{j}^{\text {pref }}\right)^{2}+\left(\partial_{y} \phi_{j}^{\text {pref }}\right)^{2}+\left(\partial_{z} \phi_{j}^{\text {pref }}\right)^{2}}$, and that for the preferred direction, $\left|\vec{\nabla} \theta_{j}^{\text {pref }}\right|=\sqrt{\left(\partial_{x} \theta_{j}^{\text {pref }}\right)^{2}+\left(\partial_{y} \theta_{j}^{\text {pref }}\right)^{2}+\left(\partial_{z} \theta_{j}^{\text {pref }}\right)^{2}}$, at position $\left(x_{j}, y_{j}, z_{j}\right)$ in the model cortex. Gradient vector components of any given function $\psi_{j}$ are given by

$$
\begin{aligned}
& \partial_{x} \psi_{j}=\frac{\partial \psi_{j}}{\partial x}=\frac{\psi\left(x_{j}+h, y_{j}, z_{j}\right)-\psi\left(x_{j}-h, y_{j}, z_{j}\right)}{2 h} \\
& \partial_{y} \psi_{j}=\frac{\partial \psi_{j}}{\partial y}=\frac{\psi\left(x_{j}, y_{j}+h, z_{j}\right)-\psi\left(x_{j}, y_{j}-h, z_{j}\right)}{2 h}, \\
& \partial_{z} \psi_{j}=\frac{\partial \psi_{j}}{\partial z}=\frac{\psi\left(x_{j}, y_{j}, z_{j}+h\right)-\psi\left(x_{j}, y_{j}, z_{j}-h\right)}{2 h}
\end{aligned}
$$

where $h$ indicates the distance between nearest neighbor model neurons, which is the size of the voxel corresponding $66 \mu \mathrm{m}$. Plotting grey spots at positions where absolute values of orientation gradient vectors are larger than $0.01 \mathrm{rad} / \mu \mathrm{m}$ and those of direction gradient vectors are larger than $0.02 \mathrm{rad} / \mu \mathrm{m}$, we obtain orientation-singularity lines and directiondiscontinuity sheets, respectively.

The positions of orientation pinwheel centers along the model cortical surface are determined automatically as the points around which the circular integration of the orientation difference amounts to just $\pm \pi$, where the circular integration is taken in the counterclockwise direction. The counterclockwise or clockwise pinwheel centers are defined by the sign of the integral. Thus-defined pinwheel centers coincided with the position of high-gradient of preferred orientations along the cortical surface. The relative number of pinwheel centers within ocular dominance subregions is analyzed according to the previously introduced method (Hübener et al. 1997). Namely, the left- or right-eye specific subregions are defined as the domains in which model neurons are classified into the top $20 \%$ of total number of neurons. The border subregions of ocular dominance are defined as the domains in which neurons exhibit binocularity within $20 \%$.

\subsection{Simulated representation of preferred orientation and direction}

We used a model visual cortex that consisted of two rectangular blocks sandwiched by two curved blocks in order to mimic the flat and curved parts of the visual cortex, respectively (Fig. $1 \mathrm{a}, \mathrm{b})$. The depth of the model cortex was 30 voxels. When we assume the depth to be $2 \mathrm{~mm}$, 
one voxel size corresponds to $66 \mu \mathrm{m}$. The rectangular blocks simulate flat parts between the lateral sulcus and the crown of the lateral gyrus, whereas the curved blocks simulate the crown of the lateral gyrus. To minimize the size effect, we imposed a periodic boundary condition on the model visual cortex. In the computer simulation, we assumed the standard deviation of the dendritic arbor of cortical neurons to be 2 voxels, which corresponds to 132 $\mu \mathrm{m}$. This implies a diameter of $264 \mu \mathrm{m}$, which falls in a plausible range for the tangential diameter of stellate cell dendritic arborization and pyramidal cell basal dendritic arborization in the visual cortex ( $290 \pm 88$ (SD) $\mu \mathrm{m}, \mathrm{n}=12$; estimated from the data shown in Sholl, 1953).
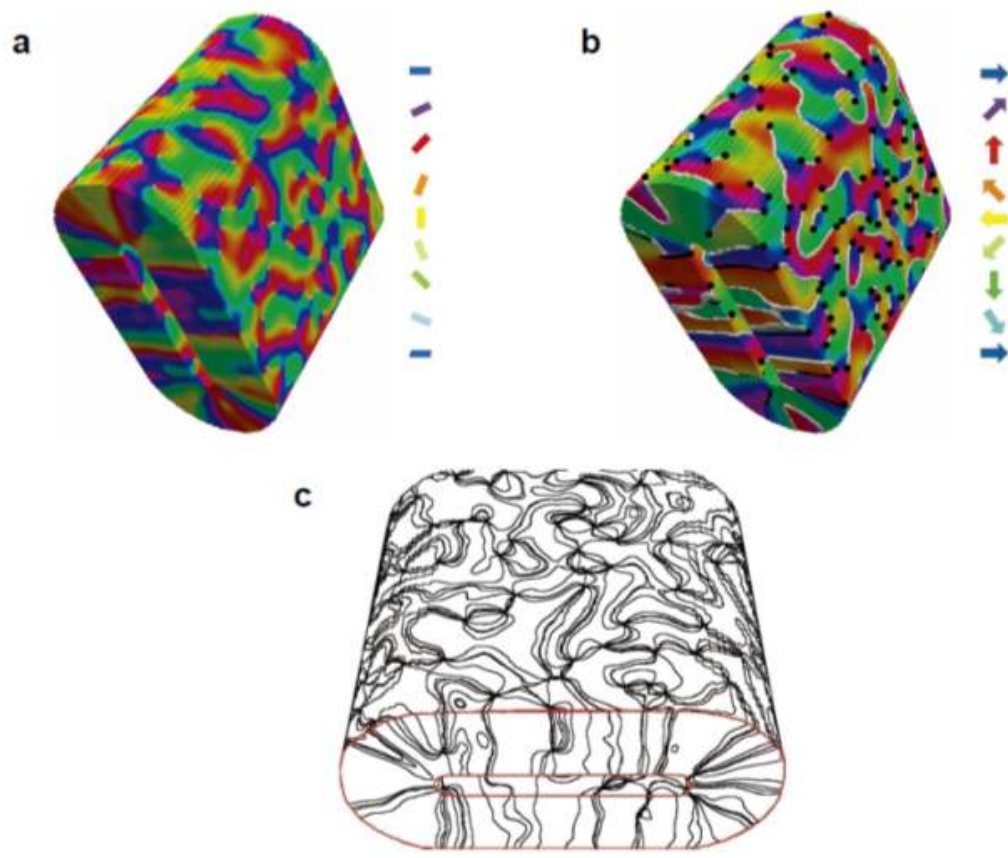

Figure 1. Three-dimensional self-organized representations.

The computer simulation formed a 3D structure of orderly arrangements of preferred orientations, which are shown by different colors (Fig. 1a). Along the model cortical surface, either in the flat or curved parts, the preferred orientation changed continuously except at singularity points called pinwheel centers, around which the preferred orientation changes in a circular fashion (Bonhoeffer \& Grinvald, 1991). The preferred orientation did not change much in the depth direction of the flat parts of the model cortex, which can be seen in the sidewall of the model cortex. This indicates that preferred orientations are arranged in a columnar fashion in the flat cortical parts. On the other hand, in the curved parts of the model cortex, preferred orientations were arranged in a wedge-like structure, where the width of orientation columns became narrower from the superficial to deeper layers. It should be noted that tips of some wedge-like columns did not reach the bottom of the model visual cortex, which corresponds to the boundary between the grey and white matter. 
Preferred directions were also arranged almost continuously along the cortical surface, except along discontinuity lines across which the preferred direction changed by $180^{\circ}$ (Fig. 1b). Such direction-discontinuity lines (Kim et al., 1999) or direction fractures (Kisvarday et al., 2001; Swindale et al., 2003) are indicated by white lines. They were terminated or branched at pinwheel centers shown as black dots in Fig. 1b, which is consistent with a previous experimental finding (Kim et al., 1999; Weliky et al., 1996) and a theoretical prediction (Tanaka, 1995, 1997). Like iso-orientation domains, iso-direction domains tended to extend vertically in the flat part of the model cortex. However, there were occasions in which the preferred direction was reversed somewhere along the cortical depth.

To more clearly visualize orientation representation particularly along the cortical depth, we illustrated iso-orientation lines (Fig. 1c), where the orientation interval between adjacent lines was $15^{\circ}$. In the flat part of the cortex, iso-orientation lines tended to penetrate throughout the cortical depth. In contrast, in the curved part, some iso-orientation lines exhibited V-shape, although some connected the cortical surface with the bottom of the cortex, forming wedge-like orientation columns.

The present model also demonstrates the emergence of ocular dominance patches mainly in the middle layer and an orderly retinotopic arrangement of the receptive field center (for more information, see Tanaka et al., 2011).

\subsection{Simulated $3 \mathrm{D}$ structures of orientation and direction singularities}

We illustrated high-gradient regions for the preferred orientation and direction. The highorientation-gradient regions appeared as thin rods, shown by gray rods in Figs. 2a and b. Note that these rods passed through pinwheel centers at any given layer. In the flat part of the cortex, $82 \%$ of high-orientation-gradient rods tended to stand straight, and the other small percent $(18 \%)$ were $\mathrm{V}$-shaped lines or hairpins. From visual inspection, all rods that reached the cortical surface intersected perpendicularly to the cortical surface (Fig. 2a). In contrast, in the curved part of the cortex, $34 \%$ of high-orientation-gradient rods appeared to be hairpins whose ends were located on the cortical surface, whereas the other percent of rods $(66 \%)$ were almost straight lines connecting the cortical surface with the bottom of the cortex (Fig. 2b). The appearance of the V-shaped orientation singularity lines is due to the fact that clockwise and counterclockwise orientation pinwheel centers in a plane parallel to the cortical surface get closer and finally they are merged to vanish, as the plane moves down from the surface to deeper layers.

On the other hand, high-direction-gradient regions appeared as thin, curved and distorted sheets, shown by gray sheets in Figs. $2 c$ and d. Figure 2c (the top view in Fig. 2a) shows the high-direction-gradient sheets in the flat part of the model visual cortex, whereas Fig. $2 \mathrm{~d}$ illustrates the side view in Fig. 2b, showing the high-direction-gradient sheets in the curved part. From visual inspection, the high-direction-gradient sheets seemed to intersect with the cortical surface or the bottom of the cortex at right angles. The intersections formed the direction-discontinuity lines on the cortical surface, which are indicated by blue lines in Figs. 2c and d (corresponding to the white lines in Fig. 1b). 
a

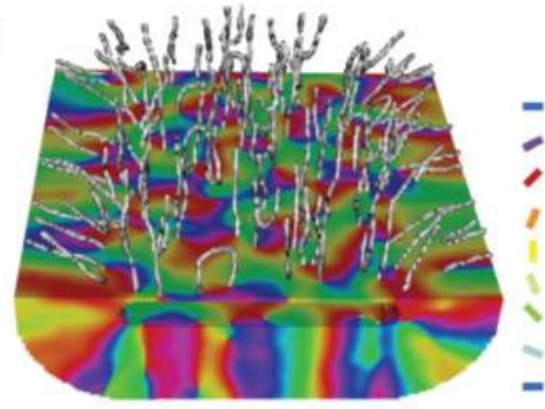

b

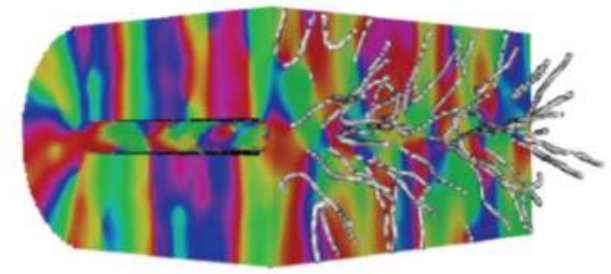

C

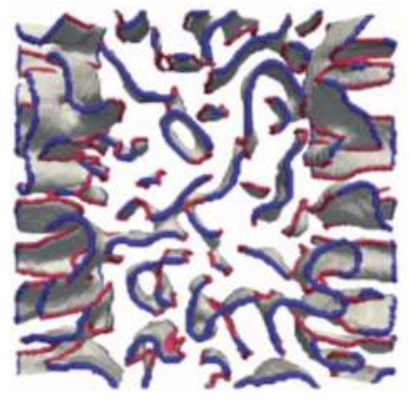

d

Top view

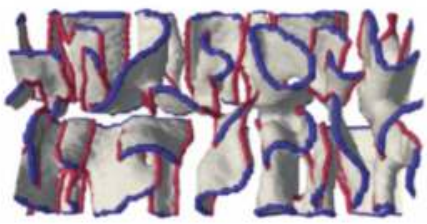

Side view

Figure 2. Orientation and direction singularities in the model cortex.

The high-direction-gradient sheets were delineated by the high-orientation-gradient rods (red rods) or the upper and lower cortical surfaces. This 3D relationship is a natural extension of the 2D relationship between pinwheel centers and direction-discontinuity lines (Figs. 1a and b). Hereafter, we refer to high-orientation-gradient rods as orientationsingularity lines and high-direction-gradient sheets as direction-discontinuity sheets. As seen in Fig. 2d, when we move radially from the cortical surface to the bottom of the cortex, the direction-discontinuity sheets became crowded. Even in the flat part (Fig. 2c), directiondiscontinuity sheets covered a non-negligible area of the cortex when we see the cortex from the top. Such patterns indicate that when an electrode penetrates vertically into the visual cortex in electrophysiological recording, the preferred direction along the electrode track can reverse somewhere in the middle of the cortical depth.

To examine how the preferred orientation and direction changed in the flat and curved parts of the model cortex, we sampled 40 traces of preferred orientations and preferred directions along the cortical depth from the surface to the bottom of the cortex perpendicularly to the cortical surface. Most traces in either the curved or flat parts showed gradual changes in the preferred orientation deviating from the orientations at the cortical surface. Some traces showed large changes of preferred orientations in the middle of the cortical depth in the curved part. The mean amplitudes of changes in the preferred orientation along the depth were $35.1^{\circ}$ and $30.5^{\circ}$, and the standard deviations of the amplitudes of changes in the preferred orientation were $35.0^{\circ}$ and $16.0^{\circ}$, in the curved and flat parts, respectively. These values indicate that in the curved cortex, the preferred orientation changes drastically in some traces but it is rather constant in the other traces, whereas in the flat cortex, the 
preferred orientation changes moderately in all traces. The preferred direction in most traces showed similar changes in the preferred orientation either in the curved or flat part. Some of them, however, showed abrupt changes by $180^{\circ}$ in the middle of the cortical depth. More reversals of preferred directions were found in the deeper layers in the curved part. We analyzed the depth-dependent probabilities to hit direction reversals in the flat and curved parts of the cortex, by sampling 14336 traces in the flat part and 14476 traces in the curved part, discarding the other traces in the transition regions between the flat and curved parts. If the direction-discontinuity sheets are flat planes, the probability to hit direction reversals is independent of the depth. However, direction reversals tended to occur more frequently midway through the cortical depth than near the cortical surface or the bottom of the cortex. This tendency reflects that direction-discontinuity sheets were wavy. The small probability to hit direction reversals near the cortical surface and the bottom of the cortex indicates that the direction-discontinuity sheets tended to intersect the cortical surface and the bottom of the cortex at right angles. In contrast, in the curved part, the preferred direction reversed more frequently in deeper layers than in superficial layers. The direction reversal in deeper layers appeared twice to three times more frequently in the curved part than in the flat part.

\subsection{Multi-slice fMRI imaging of 3D orientation representation}

To compare simulated results with the feature representation in the cat visual cortex, highresolution fMRI was performed on cat visual cortex at $9.4 \mathrm{~T}$ (Fukuda et al., 2006; Moon et al., 2007). The cat was anesthetized with isoflurane $(0.8-1.0 \%)$ and immobilized with pancuronium bromide $\left(0.2 \mathrm{mg} \mathrm{kg}^{-1} \mathrm{hr}^{-1}\right.$, i.v.). The imaging positions were selected in the region of dorsal surface and smaller surface veins, based on high-resolution 3D anatomical images. fMRI data were acquired using the multi-slice 2D gradient echo planner imaging sequence with the following parameters: $\mathrm{TR}=2.0 \mathrm{~s}, \mathrm{TE}=10 \mathrm{~ms}$, matrix $=96 \times 96$, and $\mathrm{FOV}=$ $2 \times 2 \mathrm{~cm}^{2}$, after an intravascular bolus injection of a dextran-coated monocrystalline iron oxide nanoparticles (MION) contrast agent (20 mg Fe kg-1 body weight). Slice thickness of $500 \mu \mathrm{m}$ was chosen to achieve sufficient sensitivity, and an inter-slice distance of $375 \mu \mathrm{m}$ was used to improve nominal resolution along a slice direction. Visual stimulation was given binocularly. Eight different orientations with high-contrast square-wave full-field moving gratings $(0.15 \mathrm{cpd}, 2 \mathrm{~Hz}$, movement direction reversal per $0.5 \mathrm{~s})$ were presented sequentially $\left(22.5^{\circ}\right.$ angle increments, $10 \mathrm{~s}$ per angle) during the one cycle of $80 \mathrm{~s}$. One stimulation cycle was repeated ten times continuously (i.e., total $800 \mathrm{~s}$ per run). Twenty runs were performed for signal averaging.

In the data analysis, first, we obtained single-condition maps for the 8 stimulus orientations at 8 slices according to Kalatsky \& Stryker's method (Kalatsky \& Stryker, 2003). We embedded each single-condition map into a rectangular solid region of $192 \times 192 \times 25$ voxels, each of which was the size of $104 \times 104 \times 125 \mu \mathrm{m}^{3}$. (For more information, see Supporting Figure 1 of Tanaka et al., 2011). Then we applied the 2D Gaussian filtering with the standard deviation of $193 \mu \mathrm{m}$ to the single-condition maps for 8 stimulus orientations at each slice. Then we applied the same filtering to these single-condition maps in the depth direction. The preferred orientation at each voxel was determined by the vector sum method 
(Bonhoeffer \& Grinvald, 1991), which is based on the Fourier analysis in the circular symmetric orientation dimension. Next, we calculated the orientation gradient at each voxel using Eq. (13) to show the region of orientation singularities. When we visualized highorientation-gradient regions, we used the same threshold value as in the model.

\subsection{Orientation representation reconstructed by high-resolution fMRI}

Figures $3 a$ and $b$, respectively, are top and coronal views reconstructed from a 3D venogram. Abbreviations of $\mathrm{A}, \mathrm{P}, \mathrm{L}$ and $\mathrm{R}$ for the cortical coordinate in Fig. 3a indicate anterior, posterior, left and right, respectively. Venous vessels were enhanced in the dark intensity dots and lines from tissues. The disk region of a $1.5-\mathrm{cm}$ diameter with a $3.125-\mathrm{mm}$ thickness surrounded by the red lines was selected for imaging. fMRI data were acquired using the multi-slice 2D gradient echo planar imaging sequence in $2 \times 2 \mathrm{~cm}^{2}$ around the lateral gyrus including areas 17 and 18 (Fig. 3a), following an intravascular bolus injection of MION. Eight 0.5-mm-thick slices were obtained from a 3.125-mm-thick slab (center-to-center distance of neighboring slices $=0.375 \mathrm{~mm}$ ) (Fig. 3b).

a

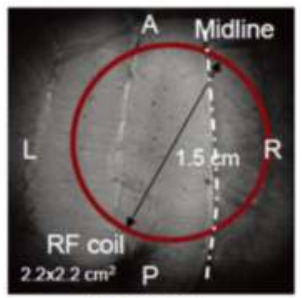

Top view

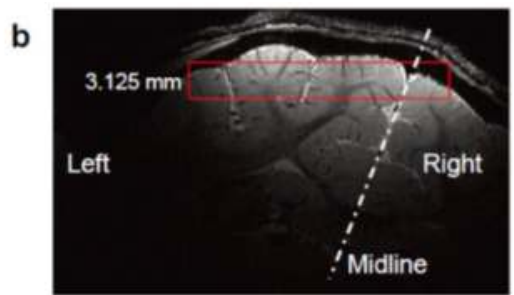

Coronal view
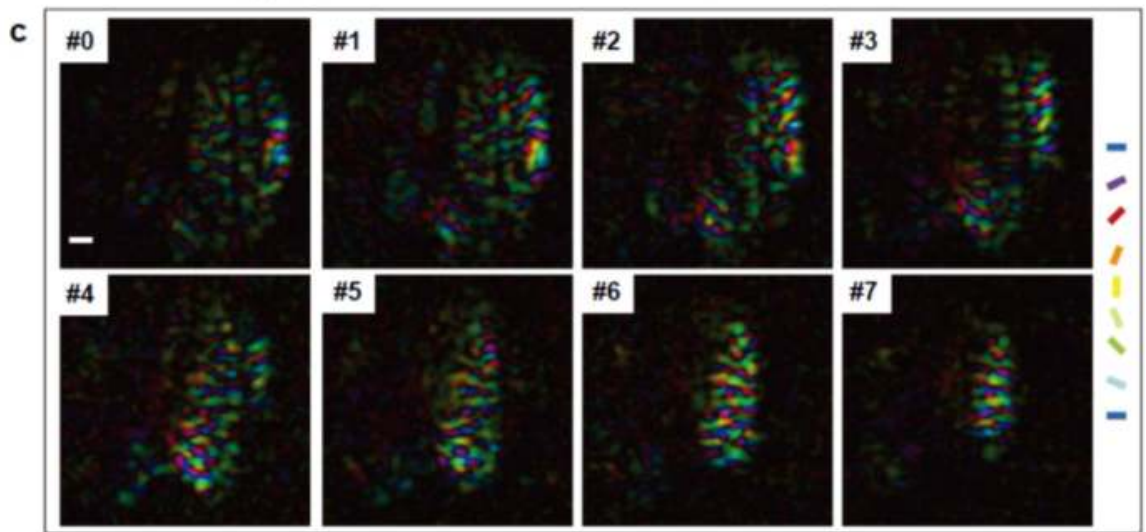

Figure 3. Multi-slice high-resolution fMRI of cat visual cortex.

Eight single-condition maps responding to binocular full-field gratings were obtained for each slice. Then, 8 orientation polar maps were calculated for different depths in the rectangular region, where color and brightness indicate the preferred orientation and orientation selectivity, respectively (Fig. 3c). The slice number is assigned from the bottom to the top in the 
imaging rectangular parallelepiped. The white scale bar placed at the bottom of slice \#0 indicates $1 \mathrm{~mm}$. Each map shows the characteristic spatial clustering of preferred orientations.

Figure 4 shows orientation representation and orientation-singularity lines in the $125-\mu \mathrm{m}$ thick slices, where the abbreviations for the cortical coordinates, M, L, D and V indicate, respectively, medial, lateral, dorsal and ventral, and all black scale bars indicate $1 \mathrm{~mm}$. In Fig. 4 in a saggital section containing the white matter, the orientation columns tended to terminate at the bottom of the grey matter at right angles with the boundary between the grey and white matter. The regions of high orientation gradient in the same section appeared as nearly straight rods, which terminated at pinwheel centers on the cortical surface (Fig. 4b). Figure 4c shows the lateral view of orientation representation in the section more medial to the section shown in Fig. 4a, which did not contain the white matter. Around the horizontal dotted line in this section, which corresponded to the bottom of the curved cortex in the medial region of recording, iso-orientation domains were not aligned vertically, different from those in Fig. 4a. Orientation-singularity lines were fractioned around there (Fig. $4 \mathrm{~d}$ ). These features imply that iso-orientation domains and singularity lines tend to run along the radial axes of the gyrus.

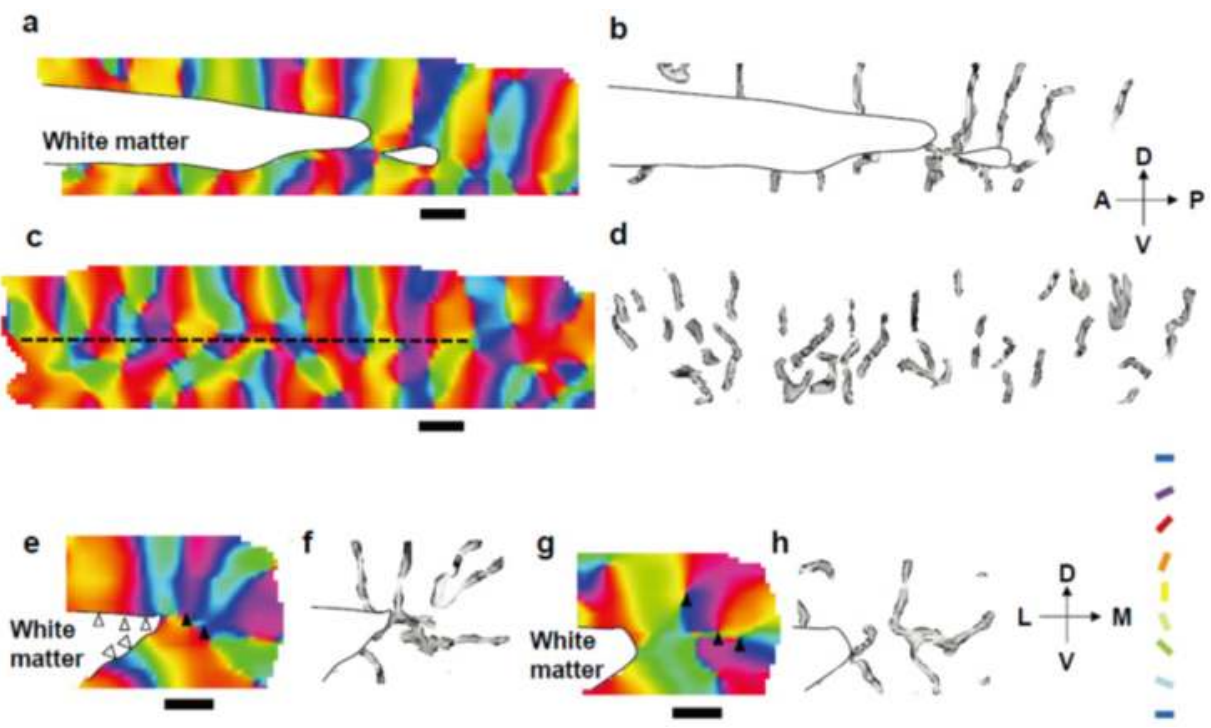

Figure 4. Orientation representations and high orientation gradients in typical sections.

In the coronal sections around the gyrus, preferred orientations were arranged in wedgelike columns rather than straight columns (Figs. 4e and g), whereas iso-orientation domains ran orthogonal to the white matter closer to the flat parts of the cortex (white arrow heads in Fig. 4e). Tips of some wedges representing single orientations (black arrow heads in Figs. 4e and g) did not reach the white matter, which indicates that orientation preferences can differ between the superficial and deep layers along the radial axes of the gyrus. Related to this feature, the orientation-singularity lines appeared as hair-pins (Figs. $4 \mathrm{f}$ and $\mathrm{h}$ ). 
To visualize the orientation columns around the curved and flat cortices more clearly, we illustrated iso-orientation lines in 3 slices of the coronal section and in 1 slice of the sagittal section, where the orientation interval between adjacent contour lines was set at $15^{\circ}$ in Fig. 5 . In the curved cortex, although some iso-orientation lines tended to reach the white matter, many lines were hairpins or converged to some points in the grey matter, and hence did not connect the cortical surface with the white matter (Figs. 5a-c). These characteristic features agree with the simulated results in the curved part of the model cortex (Fig. 1c). On the other hand, isoorientation lines tended to connect the cortical surface and the white matter straightly in the flat cortex as shown in the dotted square of Fig. 5d. Interestingly, even in the flat cortex, some isoorientation lines were hairpins or converged to points in the grey matter. This indicates that all orientation columns in the flat cortex are not necessarily straight columns.
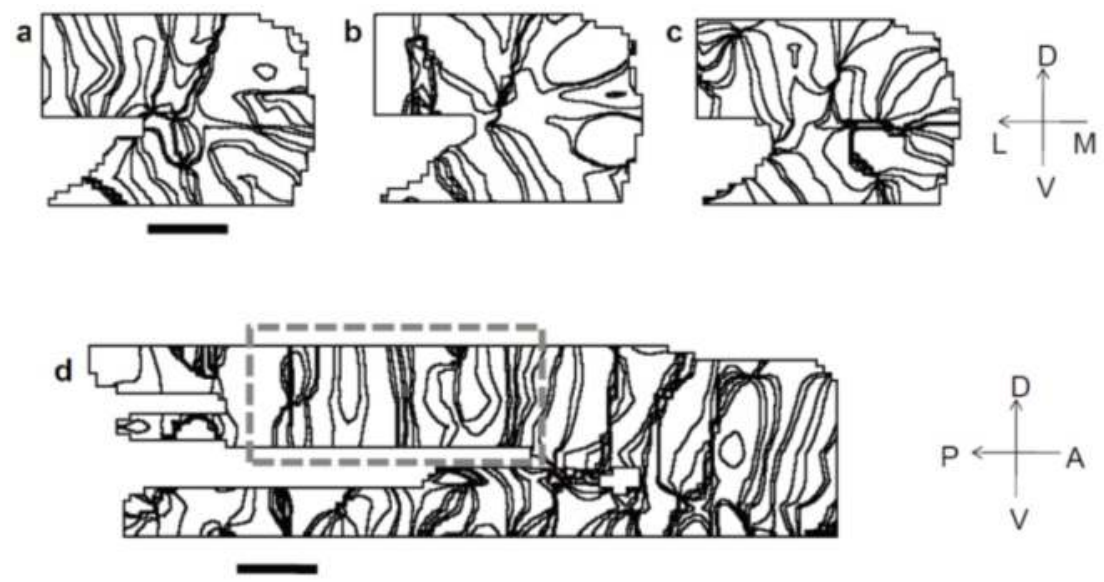

Figure 5. Iso-orientation line maps in 4 typical sections.

When the planes of the slice sections are not parallel to the straight columns that penetrate throughout the grey matter, they may appear to terminate in the middle of the cortical depth. Also, when orientation columns are wavy, even if penetrating throughout the grey matter, they may appear to terminate in the middle of the cortical depth. To examine quantitatively whether orientation columns continuously extend throughout the cortical depth at least within the imaged region, we defined the penetration index: It takes 1 when we can find a continuous path from the surface down to the white matter, keeping the same orientation within the range of $22 .^{\circ}$, and otherwise, 0 . The penetration indices were averaged in flat or curved parts both for the experimental and theoretical results. To estimate average penetration indices, we used 14336 traces $(=56 \times 128 \times 2$ pixels $)$ in the up- and downside of the flat parts and 14336 traces $(\cong[72 \times \pi / 4] \times 128 \times 2$ pixels $)$ in the most lateral curved parts in the simulated result, and 636 and 830 traces in the flat and curved parts, respectively, in the experimental result. The average penetration index estimated from the fMRI data was 0.87 for the flat cortex, and 0.78 for the curved cortex. These values indicate that not all orientation columns continued from the cortical surface to the white matter. However, some orientation columns reached the white matter, even if they may be wavy. The fact that the 
average penetration index was smaller in the curved part than in the flat part indicates that orientation columns more often terminate somewhere in the cortical depth in the curved cortex, consistent with visual inspection of Figs. 4 and 5.

On the other hand, in the simulated results, the average penetration index was 0.99 in the flat part of the cortex, and 0.89 in the curved part. It is quite likely that these values are larger than those obtained from the experiment, because detailed connectivity among different neurons and its complexity were omitted in the model. However, these two values again indicate that orientation columns in the curved cortex more frequently terminate somewhere in the cortical depth than do those in flat cortex. Taken together, the experiment and theory show that the cortical curvature partially deforms columnar organization and disrupts some columns in the middle of the cortical depth.

\subsection{Effects of curvature of visual cortex}

The cerebral cortex is packed into the skull and folded in a complex manner. Accordingly, most parts of the cerebral cortex are curved. It would be expected that sensory feature representation is constrained by the curvature of the cortex. Schematic pictures of columnar organization for feature representation shown in many textbooks (for example, see Kandel et al., 2000) may be too simple, particularly in the curved part of the gyrus. Hubel and Wiesel (1962) suggested that wedge-like columns appear in the apical segment of the postlateral gyrus because the columns are parallel to the radial fiber bundles and perpendicular to cortical layers. The present simulation and fMRI data demonstrated that orientation columns are likely to appear as wedge-like columns rather than straight columns in curved parts of the cortex.

More interestingly, the columns do not necessarily extend from the cortical surface to the white matter. Related to this property, the present studies showed that orientation singularities were not necessarily straight lines penetrating from the cortical surface to the white matter. Some of them appear as hairpins, as shown in the simulation (Figs. 2a and b) and in the fMRI data from cat visual cortex (Figs. $4 \mathrm{f}$ and $\mathrm{h}$ ). Since the topological constraint on the preferred orientation and direction should be valid in the bulk of the cortex either flat or curved, direction-discontinuity sheets are delimited by orientation-singularity lines. The original view of the configuration of orientation columns as the stacked-slab arrangement (Hubel et al., 1977) has been revised as a view of a pinwheel arrangement of preferred orientations (Bonhoeffer \& Grinvald, 1991), retaining the concept of organization of straight columns. The present study suggests that columnar organization may be more complex than expected from the conventional hypercolumn picture: orientation columns are distorted to reconcile with the global curvature of the visual cortex. The wedge-like shape of an orientation column and the interruption of columns in the deeper layers can release the tension induced by the columnar organization in curved grey matter. Our model indicates that such a structure emerges from the competition between the tendencies of periodic arrangement and radial alignment of orientation representation in a curved cortex. Because a large part of area 17 is located near the crown of the gyrus, anatomical minicolumns arranged along the radial fiber bundle may functionally represent different orientations in 
superficial layers and deep layers in area 17. Moreover, the present model predicts that the preferred direction can more frequently reverse in the deeper layers around the gyrus than in the flat cortex, as reported by Berman et al. (1987). To date, it is thought that the cortical information representation obeys a columnar organization rule. The present study suggests that careful examination of functional architecture is needed, because different pieces of information can be represented in an identical anatomical minicolumn at different layers, particularly around the crowns of gyri and perhaps at fundi.

\section{Orientation map plasticity in early life}

To investigate the visual cortical plasticity of orientation selectivity, we need to manipulate visual experience to expose animals to restricted orientations continuously and stably. However, manipulating experienced orientations is more difficult than depriving one eye by eyelid suture for the investigation of ocular dominance plasticity (Wiesel \& Hubel, 1963). For the stable orientation-restricted visual experience, we fabricated simple-structured goggles fitted with cylindrical lenses, which can be easily attached and dettached to the forhead of cats. In addition, to avoid a sampling bias problem often encountered in unit recording, we employed optical imaging of intrinsic signal to measure orientation selectivity from a wide cortical area. Some kittens were reared with head-mounted goggles to experience a single orientation in a freely moving condition inside animal cages with their mother cats and littermates for two weeks. Some other kittens were reared without goggles, while the other conditions were the same as in goggle-reared kittens. These kittens were used for a control group.

\subsection{Experimental methods}

The goggles were composed of planoconvex acrylic cylindrical lenses as shown in Fig. 6a (lens thickness, $10.0 \mathrm{~mm}$; lens aperture diameter, $15.0 \mathrm{~mm}$; lens power, +67 D). Cats on which goggles were mounted (Fig. 6b) were able to see elongated images of their environments through the goggles (Tanaka et al., 2007). For example, an image of concentric pattern is transformed to an image of vertical stripe (Figs. 6c and d). We used two types of goggles: vand h-goggles, which elongated visual images vertically and horizontally, respectively.
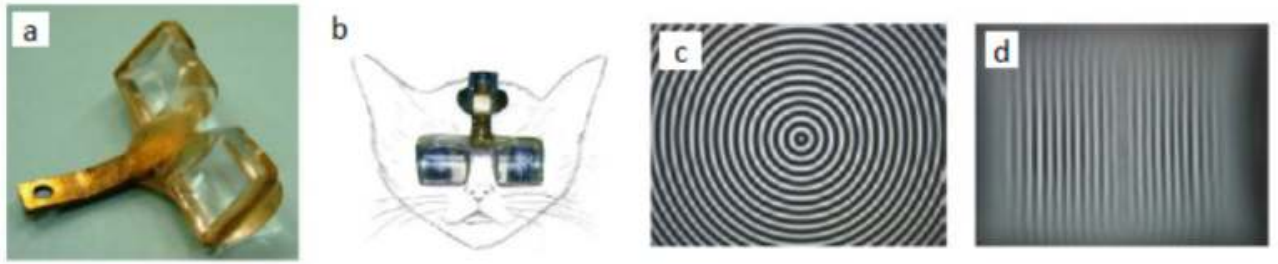

Figure 6. Cylindrical-lens-fitted goggles and visual image transformation.

Surgery was conducted according the procedure described in our previous papers (Tanaka et al., 2004, 2006). Initial anesthesia was induced using ketamine hydrochloride $(5.0 \mathrm{mg} / \mathrm{kg}$, i.m.) following sedation with medetomidine hydrochloride $(0.1 \mathrm{mg} / \mathrm{kg}$, i.m.). The animals 
were fixed on a stereotaxic apparatus and were artificially ventilated with a $60: 40 \%$ mixture of $\mathrm{N}_{2} \mathrm{O}$ and $\mathrm{O}_{2}$ containing $0.5-1.0 \%$ isoflurane. Heart rate, end-tidal $\mathrm{CO}_{2}$ concentration, and rectal temperature were continuously monitored during surgery. A metal head holder for fixing the goggles and a metal chamber for optical imaging were cemented on the animal's skull using dental resin, and the skull and dura mater covering the recording area of the lateral gyrus were removed. The cranial window $(17 \mathrm{~mm} \times 12 \mathrm{~mm})$ was positioned approximately from P5 to A12, spanning the midline. Next, the chamber was filled with $2 \%$ agar and sealed with a polyvinylidene chloride thin film and a plastic plate. Finally, the frame of the goggles was fixed to a head holder and the position of the goggles was calibrated so that the cylindrical lenses covered the visual field as widely as possible.

Animals were anesthetized as in surgery and paralyzed with pancuronium bromide $(0.1$ $\mathrm{mg} / \mathrm{kg} / \mathrm{h}$ ). They were artificially ventilated. Contact lenses with appropriate curvatures were used to prevent the drying of eyes. The cortex was illuminated with a 700-nm wavelength light. The focal plane was adjusted to $500 \mu \mathrm{m}$ below the cortical surface using a tandem-lens macroscope arrangement (Ratzlaff \& Grinvald, 1991). Intrinsic optical signals were measured while the animals were exposed to visual stimuli displayed on a 20 -inch CRT monitor placed $30 \mathrm{~cm}$ in front of the animal. Images were obtained with a CCD video camera, and digitized and stored in a computer. For each stimulus presentation, the intrinsic signal was recorded for $1.0 \mathrm{~s}$ before and $5.0 \mathrm{~s}$ after the stimulus onset. A blank stimulus was presented for $15 \mathrm{~s}$ between successive captures of intrinsic signals. Each visual stimulus was presented once in a pseudorandom sequence in a single trial of recordings. Twenty-six to 30 trials were collected in each recording session. As visual stimuli, we used full-screen squarewave gratings, which drifted in two directions at six equally spaced orientations (interval, $30^{\circ}$ ). To functionally identify area 17 , we used gratings of a spatial frequency of 0.5 c.p.d., which is optimal for area 17 neurons (Bonhoeffer et al., 1995; Movshon et al. 1978; Ohki et al., 2000). The temporal frequency of the gratings was fixed at $2.0 \mathrm{~Hz}$. The optical imaging in one session was completed within 5 hours.

The analysis methods that we used were described in a previous paper (Tanaka et al., 2006). It is noteworthy to explain the methods in some detail here to show that observed map changes are attributable to biological changes rather than artificial changes originating from our analysis methods. One trial of optical imaging was composed of six frames (duration of each frame, $1 \mathrm{~s}$ ). To extract stimulus-related intrinsic signals, we subtracted signals recorded in the first frame (without stimulus presentation) from those signals recorded in succeeding frames with stimulus presentations. Then, we averaged the subtracted signals over the 4th to 6th frames for each trial. Next, we applied the generalized indicator function method to these averaged signals (Yokoo et al., 2001), which efficiently excluded noisy signals originating from volume and oxygenation changes in thick blood vessels and spatially slowly varying fluctuations of signals inherent in the recorded intrinsic signals. It should be noted that the image data processing based on the generalized indicator function method underestimates the effects of overrepresentation of exposed orientation induced by singleorientation exposure, because the data processing method eliminates spatially slowly varying point-spread components of intrinsic signal (Gilbert et al., 1996), which may partially contain responses to the exposed orientation. 
Having excluded the spatially slow noise components, we summed the stimulus-related signals over all trials for each stimulus orientation and applied Gaussian low-pass filtering with a $150-\mu \mathrm{m}$ standard deviation to eliminate high-frequency noise. In this way, we constructed a single-condition map for each stimulus orientation. To determine the preferred orientation at each pixel inside the recorded area, we used the vector sum method (Bonhoeffer \& Grinvald, 1991). Thus, at each pixel, we obtained the preferred orientation and the modulation amplitude in the second harmonic components, which is regarded as orientation selectivity. The orientation polar map was constructed with the preferred orientation and orientation selectivity as color and brightness, respectively.

For further analysis, we discarded pixels eliciting response strengths lower than a half of the response strength averaged over all pixels inside the recorded area. According to this criterion, the domains containing the remaining pixels nearly lined up with functionally defined area 17 , which was exclusively activated by stimuli of a 0.5-c.p.d. spatial frequency. To construct an orientation histogram, we counted the number of pixels involved in each orientation, $30^{\circ}$ width, and normalized them by the total number of pixels involved in all orientations.

\subsection{Orientation map alteration by goggle rearing}

Before examining orientation maps in goggle-reared cats, we investigated orientation maps in normally reared cats. In orientation polar maps obtained from normally reared kittens younger than P30, generally the relative size of responsive domains is largest for horizontal orientation $\left(0^{\circ}\right.$ or equivalently $\left.180^{\circ}\right)$ and smallest for vertical orientation $\left(90^{\circ}\right)$. This indicates that orientation representation is biased toward the horizontal orientation for very young normal kittens. This is analogous to the innate bias toward the contralateral eye, as has been known in ocular dominance (Wiesel \& Hubel, 1963). However, the sample-averaged orientation histogram across 15 normally reared kittens of P33-84 showed a weak bias toward vertical orientation.

Here, we first describe the results of goggle rearing (GR) for two weeks. Measurements in goggle-reared kittens were completed within $5 \mathrm{hrs}$ after the removal of the goggles. Figure 7 shows orientation polar maps (a, c, e, g, i and k) and orientation histograms (b, d, f, h, j and l) obtained from optical imaging of functionally defined area 17 (delineated by white curves) of those kittens. In orientation polar maps, color and brightness indicate preferred orientation and orientation selectivity, respectively (scale bars: $2 \mathrm{~mm}$ ), and in orientation histograms, the height of bins indicates the relative size of cortical domains for 6 preferred orientations, where blue horizontal lines indicate the relative size of iso-orientation domains for a uniform orientation representation. Immediately after the 2-week GR that started at P16-P17, the overrepresentation of horizontal orientation was exclusive (Figs. $7 \mathrm{~g}$ and $\mathrm{h}$ ), whereas that of vertical orientation was somewhat moderate (Figs. 7a and b). For GR that started at P27-P29, the overrepresentation of vertical orientation also became nearly exclusive (Figs. 7c and $d$ ). The representation of horizontal orientation was still predominant, although that of unexposed orientations appeared (Figs. $7 \mathrm{i}$ and j). The imbalance between vertical and horizontal orientations at P16-P17 may reflect, at least partly, the horizontal bias detected in normal kittens. When 2-week GR was started at P49-54, the induced overrepresentation of the exposed 
orientation, either vertical or horizontal, diminished drastically (Figs. 7e, f, k and l). A close examination of Figs. 7e and $f$ revealed that the effect of vertical GR was reversed to a slight underrepresentation of the vertical orientation.
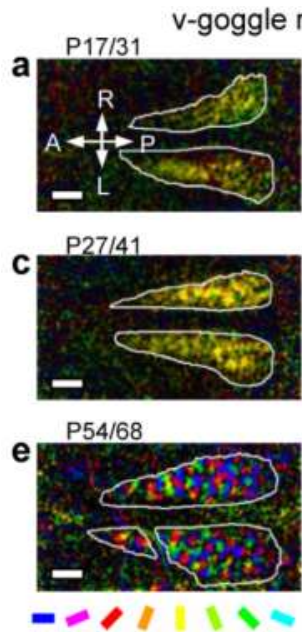

Orientation polar maps
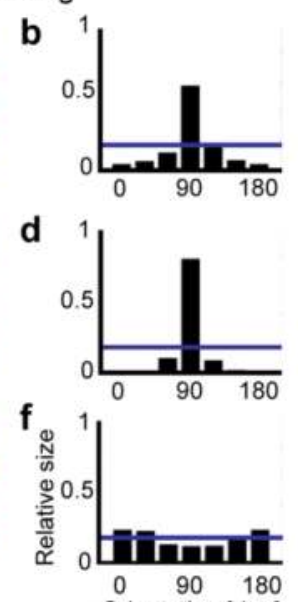

Orientation [deg] Orientation histograms

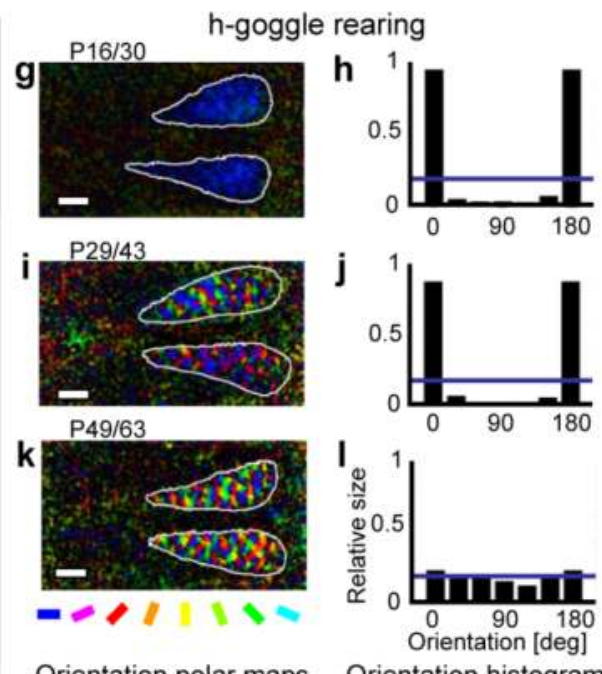

Orientation polar maps

Orientation histograms

Figure 7. Orientation selectivity in kittens continuously exposed to vertical- or horizontal orientation for 2 weeks.

\subsection{Critical period for orientation selectivity}

Figure 8 illustrates the time profile of the sensitivity for the modification of orientation selectivity against the onset day of GR, where all data points were obtained from 18 kittens goggle-reared for 2 weeks. Here, we quantified the sensitivity as the relative size of the cortical domains for the exposed orientation, in which the average representation bias in normal kittens was subtracted at respective days of optical imaging performance (for more information, see Tanaka et al. 2007). Therefore, the positive and negative normalized relative domain size indicate the overrepresentation and underrepresentation of the exposed orientation, respectively. The sensitivity profiles for vertical- and horizontal-orientation exposures are generally consistent with each other, except for a discrepancy at 2-3 postnatal weeks. This discrepancy suggests that innate mechanisms of orientation map formation not only induce the representation bias toward horizontal orientation in normal kittens but also enhance the overrepresentation of horizontal orientation in young kittens exposed to horizontal orientation. The critical period can be defined as the postnatal period during which 2-week GR effectively causes the modification of orientation selectivity. Thus defined, the critical period starts 2 weeks after birth and lasts for 6 weeks. Note that the presently delineated critical period for orientation plasticity overlaps the most sensitive period for ocular dominance plasticity (postnatal 4-5 weeks) reported by Olson \& Freeman (1980). 


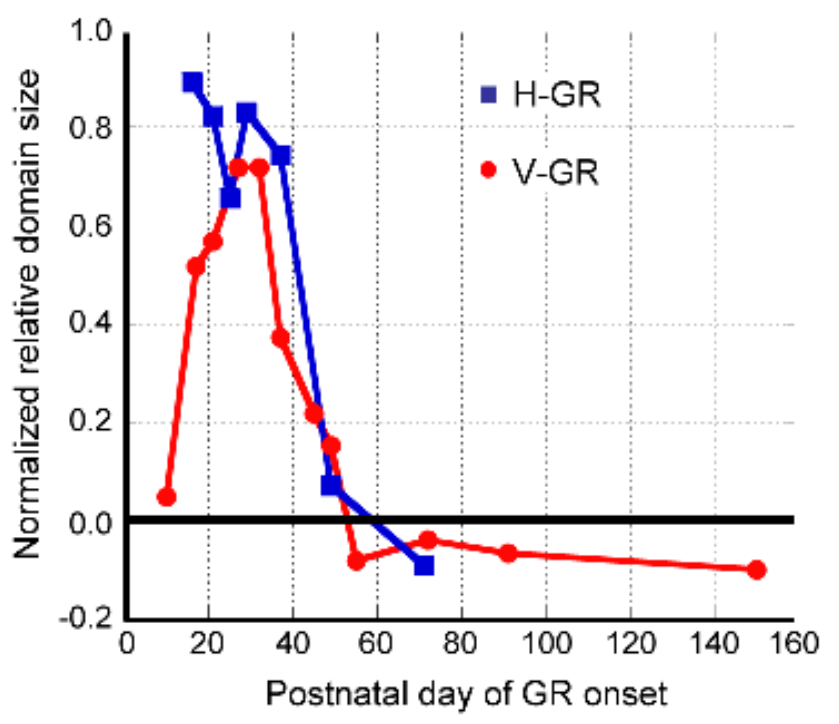

Figure 8. Sensitivity profiles for orientation plasticity.

Figure 8 also shows that the critical period is followed by a late phase of underrepresentation of the exposed orientation for GR starting between P55 and P151. We also observed the case of an adult cat reared with vertical goggles from P396 for one month; this cat showed a small relative size of cortical domains representing the exposed orientation for $90^{\circ}$. Therefore, continuous single-orientation exposure after the critical period, even in adulthood, leads to the underrepresentation of the exposed orientation, as consistent with previous reports on orientation plasticity in adult cats (Creutzfeldt \& Heggelund, 1975; Dragoi et al., 2000).

\subsection{Orientation map alteration under short- and long-term GR}

Figure 9 shows how the once-induced overrepresentation of the exposed orientation changes afterwards. The normalized relative sizes of cortical domains representing exposed vertical (circles) or horizontal (squares) orientations are plotted at the end of 2-week GR (solid symbols) and also at the end of the succeeding normal viewing (hollow symbols), respectively. Plotted points for identical kittens are linked by the lines, and numerical figures indicate onset ages of GR. In 4 kittens for which 2-week GR started at P29, 32, 37 and 39, 3-day normal viewing immediately before the second optical imaging eliminated the overrepresentation: the normalized relative domain sizes returned to 0 (level of normal kittens). However, in 4 kittens in which 2-week GR started relatively earlier at P16, P21 and P25, recovery during the succeeding 3-days of normal viewing was partial. 


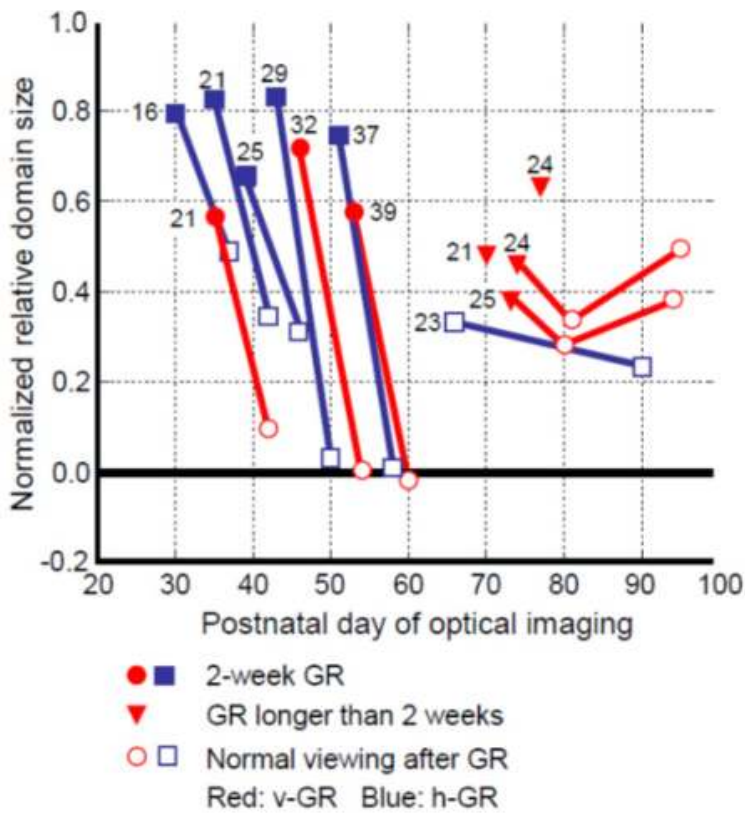

Figure 9. Effects of duration and timing of GR.

In 4 other kittens, in which long-term vertical GR for 4-6 weeks started at P21-P25 (triangles), the overrepresentation of the exposed orientation was retained at moderate levels between 0.39 and 0.64 of the normalized relative domain size in the first optical imaging. As tested in two of these kittens (P24-P73; P25-P74), the overrepresentation was preserved even after 3 weeks of normal viewing. A similar tendency was observed in another kitten in which horizontal GR started at P23 and switched to normal rearing at P51.

We can summarize normal viewing effects on GR-induced changes of orientation maps as follows:

1. When 2-week GR covers a relatively late phase of the critical period (at P29-39), normal viewing quickly eliminates the overrepresentation and restores normal orientation maps.

2. When 2-week GR covers a relatively early phase of the critical period (earlier than P29), normal viewing does not completely eliminate the overrepresentation.

3. Goggle rearing for 4 weeks or more outlasting the critical period acts to preserve the moderate overrepresentation of the exposed orientation, and reorganized orientation maps are consolidated to be robust against normal viewing thereafter.

\subsection{Comparisons with previous studies}

There have been two hypotheses about the effect of visual experience on orientation plasticity. One is the selection hypothesis and the other the instruction hypothesis. In the 
former hypothesis, after single-orientation exposure, neurons innately selective for unexposed orientations just decrease their responses to the unexposed orientations without changes of preferred orientations. In the latter hypothesis, neurons selective for unexposed orientations change their preferred orientations towards the exposed orientation. Blakemore \& Cooper (1970) supported the instruction hypothesis, because they found preferred orientations of recorded units were strongly biased toward the exposed orientation in cats that had experienced striped environment. Later, Stryker et al. (1978) found that singleorientation exposure changed a large portion of units nonselective or unresponsive, although a proportion of responsive units preferring for the exposed orientation increased. Particularly, they found an orderly arrangement of selective units according to preferred orientation along the electrode tracks, as observed in normal cats, but clusters of nonselective or unresponsive units were interleaved. In our optical imaging on kittens exposed to a single orientation, stimulus-related intrinsic signals in response to unexposed orientations were reduced in cortical domains originally selective for the unexposed orientations, and a proportion of pixels without orientation selectivity tended to increase (Tanaka et al., 2006), consistently with single-unit recording by Stryker et al. (1978). However, stimulus-related intrinsic signals in response to the exposed orientation tended to increase in these domains, resulting in the changes of orientation preference.

Differences of experienced patterns during single-orientation exposure may be worth noting. Hirsch \& Spinelli (1970) and Stryker et al. (1978) exposed kittens to stationary lines through their goggles. Carlson et al. (1986) also presented stationary stripe patterns with various spatial frequencies to monocularly deprived infant monkeys. To examine the effect of exposure to stationary oriented stimuli, we have reared 4 kittens with spherical-lens-fitted goggles for chronic exposure to a stationary stripe with a spatial frequency of about 0.5 and 0.15 c.p.d (Tanaka et al., 2007). Although the exposed orientation was overrepresented at the first optical imaging experiments after 2- or 3-week GR in 3 kittens, the underrepresentation of the exposed orientation occurred in the other kitten, in which the orthogonal orientation was overrepresented (data not shown). Even in the kittens showing the overrepresentation of the exposed orientation, the layouts of orientation preferences were labile during prolonged GR. In 3 of the 4 kittens, the overrepresentation disappeared or changed to the underrepresentation after long-term GR. Such labile alteration of orientation maps is characteristic of exposure to a stationary oriented pattern. This is contrasted with the finding that orientation maps altered by exposure to a dynamic single orientation through cylindrical-lens-fitted goggles are consolidated preserving the moderate overrepresentation of the exposed orientation (Fig. 9). It should be noted that the instability in orientation map alteration for stationary stripe pattern exposure was not due to the repeated optical imaging, because orientation maps altered by rearing with cylindrical-lens-fitted goggles changed gradually in successive optical imaging, and were finally stabilized at the moderate overrepresentation of the exposed orientation. The fact that Carlson et al. (1986) recorded units selective for the orthogonal orientation to the exposed orientation in the open eye may be such labile modification of orientation selectivity induced by the stationary stripe pattern exposure. The disappearance of the orientation selectivity modification for prolonged 
exposure to stationary oriented stimuli is suggested to have a weak impact on the structural modification of orientation maps.

The different effects of dynamic and stationary stripe exposures on orientation map alteration may be attributed to the differences in the behavioral significance of visual images through the goggles. Images transmitted through cylindrical leses contain some information on the animal's environment, in that vertically elongated stripes move associated with the movement of visual objects in the environment. It is, therefore, expected that the animal may pay attention to the images and neurons in the primary visual cortex can be sufficiently activated. Such neuronal activation consequently may have an influence on the suceptivility for orientation plasticity. In contrast, stripes exposed to by opaque lenses do not move irrespect of any dynamic changes of the environment. The animal may neglect the stripes and neurons in the primary visual cortex may not be sufficiently activated, resulting in weak or no induction of orientation plasticity. How the behavioral significance of exposed visual images affects orientation plascity is an interesting future research target.

\section{Conclusion}

Hubel \& Wiesl's hypercolumn model successfully provides a simple view of the functional architecture of the cerebral cortex. However, our simulation of visual feature representation together with the high-resolution fMRI experiment on the cat threw a doubt about the idea of orientation columns extending from pia to white matter, especially in regions where the cortex is curved. Futhermore, the dimensionality of singularities in the orientation and direction maps was inceased by 1 , when we considered the 3D visual cortex: Pinwheel centers in the orientation map appeared as point terminals of line singularities running in the 3D visual cortex at the cortical surface; direction-discontinuity lines starting or ending at pinwheel centers appeared as line crossings of direction-discontinuity sheets with the cortical surface. More extensive experiments are desired to confirm these observations.

In regrad to orientation plasticity in the developing visual cortex, it seems to be accepted that preferred orientations of neurons do not change but only responsiveness changes depending on visual experience. Our optical imaging experiments on cats, however, demonstrated that short-term single-orientation exposure dramatically altered preferred orientations until postnatal 6 weeks, which is against the current consensus. This study, on the other hand, revealed further complexity in orientation plasticity in case where animals were exposed to a single orientation for a long time or returned to a normal visual environment after single-orientation exposure. Again on this issue, further investigation is needed to obtain a better understanding of mechanisms underlying orientation plasticity in the visual cortex.

\section{Author details}

Shigeru Tanaka

The University of Electro-Communications, Japan 


\section{References}

Albus, K., \& Wolf, W. (1984). Early post-natal development of neuronal function in the kitten's visual cortex: A laminar analysis. Journal of Physiology, Vol. 348, pp. 153-185

Berman, N. E., Wilkes, M. E., \& Payne, B. R. (1987). Organization of orientation and direction selectivity in areas 17 and 18 of cat cerebral cortex. Journal of Neurophysiology, Vol. 58, pp. 676-699

Blakemore, C., \& Cooper, G. F. (1970). Development of the brain depends on visual environment. Nature, Vol. 228, pp. 477-478

Blakemore, C., \& Van Sluyters, R. C. (1975). Innate and environmental factors in the development of the kitten's visual cortex. Journal of Physiology,Vol. 248, pp. 663-716

Bonhoeffer, T., \& Grinvald, A. (1991). Iso-orientation domains in cat visual cortex are arranged in pinwheel-like patterns. Nature, Vol. 353, pp. 429-431

Bonhoeffer, T., Kim, D-S., Malonek, D., Shoham, D., \& Grinvald, A. (1995). Optical imaging of the layout of functional domains in area 17 and across the area 17/18 border in cat visual cortex. European Journal of Neuroscience, Vol. 7, pp. 1973-1988

Carlson, M., Hubel, D. H., \& Wiesel, T. N. (1986). Effects of monocular exposure oriented lines on monkey striate cortex. Developmental Brain Research, Vol. 25, pp. 71-81

Crair, M. C., Gillespie, D. C., \& Stryker, M. P. (1998). The role of visual experience in the development of columns in cat visual cortex. Science, Vol. 279, pp. 566-570

Creutzfeldt, O. D., \& Heggelund, P. (1975). Neural plasticity in visual cortex of adult cats after exposure to visual patterns. Sicence, Vol. 188, pp. 1025-127

Dragoi, V., Sharma, J., \& Sur, M. (2000). Adaptation-induced plasticity of orientation tuning in adult visual cortex. Neuron, Vol. 28, pp. 287-298

Fukuda, M., Moon, C. H., Wang, P., \& Kim, S. G. (2006). Mapping iso-orientation columns by contrast agent-enhanced functional magnetic resonance imaging: reproducibility, specificity, and evaluation by optical imaging of intrinsic signal. Journal of Neuroscience, Vol. 26, pp. 11821-11832

Gilbert, C. D., Das, A., Ito, M., Kapadia, M., \& Westheimer, G. (1996). Spatial integration and cortical dynamics. Proceedings of National Academy of Science USA, Vol. 93, pp. 615-622

Hirsch, H. V., Spinelli, D. N. (1970). Visual experience modifies distribution of horizontally and vertically oriented receptive fields in cats. Science, Vol. 168, pp. 869-871

Hubel, D. H., \& Wiesel, T. N. (1962). Receptive fields, binocular interaction and functional architecture in the cat's visual cortex. Journal of Physiology, Vol. 160, pp. 106-154

Hubel, D. H., \& Wiesel, T. N. (1963a). Shape and arrangement of columns in cat's striate cortex. Journal of Physiology, Vol. 165, pp. 559-568

Hubel, D. H \& Wiesel, T. N. (1963b). Receptive fields of cells in striate cortex of very young, visually inexperienced kittens. Journal of Neurophysiology, Vol. 26, pp. 994-1002

Hubel, D. H., Wiesel, T. N. \& LeVay, S. (1977). Plasticity of ocular dominance columns in monkey striate cortex. Philosophical Transactions of the Royal Society London B: Biological Sciences, Vol. 278, pp. 377-409

Hübener, M., Shoham, D., Grinvald, A. \& Bonhoeffer, T. (1997). Spatial relationships among three columnar systems in cat area 17. Journal of Neuroscience, Vol. 17, pp. 9270-9284 
Kalatsky, V. A. \& Stryker, M. P. (2003). New paradigm for optical imaging: temporally encoded maps of intrinsic signal. Neuron, Vol. 38, pp. 529-545

Kandel, E. R., Schwartz, J. H. \& Jessell, T. M. (2000). Principles of Neural Science (4th edition), McGraw-Hill, 978-0838577011, New York

Kim, D. S., Matsuda, Y., Ohki, K., Ajima, A. \& Tanaka, S. (1999). Geometrical and topological relationships between multiple functional maps in cat primary visual cortex. Neuroreport, Vol. 10, pp. 2515-2522

Kisvarday, Z. F., Buzas, P. \& Eysel, U. T. (2001). Calculating direction maps from intrinsic signals revealed by optical imaging. Cerebral Cortex, Vol. 11, pp. 636-647

Moon, C. H., Fukuda, M., Park, S. H. \& Kim, S. G. (2007). Neural interpretation of blood oxygenation level-dependent fMRI maps at submillimeter columnar resolution. Journal of Neuroscience, Vol. 27, pp. 6892-6902

Movshon, J. A., Thompson, I. D., \& Tolhurst, D. J. (1978). Spatial and temporal contrast sensitivity of neurones in areas 17 and 18 of the cat's visual cortex. Journal of Physiology, Vol. 283, pp. 101-120

Nakagama, H. \& Tanaka, S. (2004). Self-organization model of cytochrome oxidase blobs and ocular dominance columns in the primary visual cortex. Cerebral Cortex, Vol. 14, pp. 376-386

Ohki, K., Matsuda, Y., Ajima, A., Kim, D-S., \& Tanaka, S. (2000). Arrangement of orientation pinwheel centers around area 17/18 transition zone in cat visual cortex. Cerebral Cortex, Vol. 10, pp. 593-601

Olson, C. R., \& Freeman, R. D. (1980). Profile of the sensitive period for monocular deprivation in kittens. Experimental Brain Research, Vol. 39, pp. 17-21

Ratzlaff, E. H., \& Grinvald, A. (1991). A tandem-lens epifluorescence macroscope: hundredfold brightness advantage for wide-field imaging. Journal of Neuroscience Methods, Vol. 36, pp. $127-137$

Rauschecker, J. P., \& Singer, W. (1981). The effects of early visual experience on the cat's visual cortex and their possible explanation by Hebb synapses. Journal of Physiology, Vol. 310, pp. 215-239

Sengpiel, F., Stawinski, P., \& Bonhoeffer, T. (1999). Influence of experience on orientation maps in cat visual cortex. Nature Neuroscience, Vol. 2, pp. 727-732

Sholl, D. A. (1953). Dendritic organization in the neurons of the visual and motor cortices of the cat. Journal of Anatomy, Vol. 87, pp. 387-406

Stryker, M. P., Sherk, H., Leventhal, A. G., \& Hirsch, H. V. (1978). Physiological consequences for the cat's visual cortex of effectively restricting early visual experience with oriented contours. Journal of Neurophysiology, Vol. 1, pp. 896-909

Swindale, N. V., Grinvald, A. \& Shmuel, A. (2003). The spatial pattern of response magnitude and selectivity for orientation and direction in cat visual cortex. Cerebral Cortex, Vol. 13, pp. 225-238

Tanaka, S. (1995). Topological analysis of point singularities in stimulus preference maps of the primary visual cortex. The Proceedings of the Royal Society London B: Biological Science, Vol. 261, pp. 81-88

Tanaka, S. (1997). Topology of visual cortex. FORMA, Vol. 12, pp. 101-106 
Tanaka, S., Ribot, J., \& Miyashita, M. (2004). Roles of visual experience and intrinsic mechanism in the activity-dependent self-organization of orientation maps: Theory and experiment. Neural Networks, Vol. 17, pp. 1363-1375

Tanaka, S., Ribot, J., Imamura, K., \& Tani, T. (2006). Orientation-restricted continuous visual exposure induces marked reorganization of orientation maps in early life. NeuroImage, Vol. 30, pp. $462-477$

Tanaka, S., Tani, T., Ribot, J., \& Yamazaki, T. (2007). Chronically mountable goggles for persistent exposure to single orientation. Journal of Neuroscience Methods, Vol. 160, pp. 206-214

Tusa, R. J., Palmer, L. A., \& Rosenquist, A. C. (1978). The retinotopic organization of area 17 (striate cortex) in the cat. Journal of Comparative Neurology, Vol. 177, pp. 213-235

Wiesel, T. N., \& Hubel, D. H. (1963). Single-cell responses in striate cortex of kittens deprived of vision in one eye. Journal of Neurophysiology, Vol. 26, pp. 1003-1017

Yokoo, T., Knight, B. W., \& Sirovich, L. (2001). An optimization approach to signal extraction from noisy multivariate data. NeuroImage, Vol. 14, pp. 1309-1326 